\title{
Falsche Lösungsvorschläge für ein ernstzunehmendes Problem
}

\author{
Jürg Schlup \\ Dr. med., Präsident der FMH
}

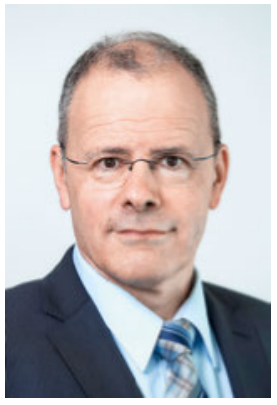

Der Anteil der Gesundheitskosten am BIP beträgt in der Schweiz 11,1 Prozent und ist damit gleich hoch wie in den Niederlanden (11,1\%), Deutschland (11,0\%), Schweden (11,0\%) und Frankreich (10,9\%). ${ }^{1}$ Gleichzeitig gehört unsere Patientenversorgung im europäischen Vergleich zu denen mit der besten Zugänglichkeit und den besten Outcomes. ${ }^{2}$ Zudem haben die Schweizer, neben Luxemburgern und Norwegern, die mit Abstand höchsten verfügbaren Einkommen. ${ }^{3}$ Zwar hat sich unsere monatliche Durchschnittsprämie zwischen 2006 und 2014 um 100 Franken gesteigert - der monatliche Durchschnittskonsum (+325 Franken), die Steuerbelastung (+370 Franken) und sogar die monatlichen Ersparnisse (+715 Franken) steigerten sich jedoch weit mehr. ${ }^{4}$

1 Die OECD in Zahlen und Fakten 2015-2016.

2 European Health Consumer Index 2016.

3 Swissinfo.ch; 25.4.2016,

Matthew Allen, Der

Lebensstandard bleibt

hoch in der Schweiz.

4 Helsana-Report, Dezem-

ber 2016. Ausgabenent-

wicklungen in der

Gesundheitsversorgung,

S. 10

5 Z.B. Tages-Anzeige

29.1.2017, Was ein Leben

kosten darf: Das Di-

lemma der Mediziner.

6 Z.B. Watson, 1.2.2017,

Das Schweizer Gesund-

heitssystem steht vor

dem Kollaps; Aargauer

Zeitung, 2.2.2017, Verdop-

pelung der Prämien-Kos-

ten: Unserem Gesund-

heitssystem droht de Kollaps.

7 16.3987 Motion: OKP

Kostenanstieg dämpfen

durch geeignete Kosten-

begrenzungs-Massnah-

men.

8 17.402 Parlamentarisch

Initiative: Steuerung de

Kosten im KVG durch

Vertragspartner.

9 Medienmitteilung des

EDI vom 2.9.2016:

Mengenwachstum im

Gesundheitswesen ein dämmen.

1017.401 Parlamentarisch

Initiative: Tarifpflege

und Entwicklung.
Warum merke ich das an? Weil mir sachliche Analyse geboten scheint - nach den Rationierungsforderungen ${ }^{5}$ samt prognostiziertem Versorgungskollaps ${ }^{6}$ der letzten Wochen. Selbstverständlich gilt es ständig zu prüfen, wo es ungenutzte Effizienz-, Verbesserungs- und Sparpotentiale gibt. Leider gehen aber die neuesten politischen Vorstösse an solch entscheidenden Fragen vorbei. - So fordert die Motion 16.3987, «dass zwingende Kostenbegrenzungsmassnahmen in Kraft treten» sollen, sobald der OKP-Kostenzuwachs (also die Prämienentwicklung) die Nominallohnentwicklung um das 1,1-fache übersteigt. ${ }^{7}$ Welche Leistungen bei welchen Patienten mit diesem reduzierten Budget nicht mehr bezahlt würden, lässt der Vorstoss offen.

- Die parl. Initiative 17.402 verlangt, dass Leistungserbringer und Versicherer fortan «in den Tarifverträgen Massnahmen zur Steuerung der Kosten und Leistungen» vorsehen, über die der Bundesrat zudem "Grundsätze» aufstellen kann ${ }^{8}$ - eine elegante Verklausulierung für die Einführung eines Globalbudgets. Der Vorstoss lässt die Inspirationsquellen des EDI - die Niederlande und Deutschland ${ }^{9}$ - erkennen. Welche Kostenvorteile diese Länder der Schweiz gegenüber aufweisen, sehen Sie oben: keine.

- Ausserdem soll der Bundesrat gemäss der parl. Initiative 17.401 zukünftig eine Tarifpflege-Organisa- tion konzipieren und sie auch selber einsetzen können. Dennoch scheint man von dieser Organisation nicht allzu viel zu erwarten. Denn trotzdem soll der Bundesrat zukünftig die Tarife und Preise selber festsetzen können, die dafür nötigen Daten sollen ihm die Leistungserbringer und Versicherer zur Verfügung stellen müssen. ${ }^{10}$

Gemeinsam ist diesen Vorstössen nicht nur die Forderung weitreichender Staatskompetenzen im Gesundheitswesen bei völliger Unklarheit der Auswirkungen auf die Patientenversorgung. Wenig rational erscheint auch, wie sich die Politik am ambulanten Arzt- und Spitaltarif TARMED geradezu festgebissen hat: Über den TARMED werden rund 11 von 71 CHF Milliarden - also ein gutes Siebtel unserer Gesundheitskosten - abgerechnet. Eine staatliche Steuerung und Deckelung dieses kostengünstigen Siebtels kann kein Kostenproblem lösen - eher würden neue geschaffen, z.B. wenn Patienten (und Ärzte) von der ambulanten in die teurere stationäre Versorgung ausweichen.

Ist es Aufgabe des Bundes, den ambulanten Arzt- und Spitaltarif zu entwickeln? Ist es zielführend, im BAG eine neue Kostenverwaltungs- oder gar Rationierungsabteilung zu schaffen? Ich denke nicht.

\section{Eine Steuerung und Deckelung von 15\% der} Gesundheitsausgaben verbessert weder Kostenentwicklung noch Lastenverteilung - schafft aber Versorgungsprobleme.

Wenn für viele Haushalte die Prämien heute ein Problem sind, obwohl die Gesamtkosten volkswirtschaftlich tragbar sind, muss der Bund die Prämienzahler entlasten, indem er die Finanzierung ambulanter und stationärer Leistungen vereinheitlicht. Damit könnte er ohne Einbussen in der medizinischen Versorgung die Prämienentwicklung bremsen, weil durch konsequenteres "ambulant vor stationär» die Patientenbehandlung günstiger würde. Hingegen die medizinische Versorgung einzuschränken, weil die Verteilung der Kostenlast nicht mehr zeitgemäss ist, wäre die falsche Lösung für ein ernstzunehmendes Problem. 